\title{
miR-218 inhibits the migration and invasion of glioma U87 cells through the Slit2-Robo1 pathway
}

\author{
JIAN-JUN GU ${ }^{1 *}$, GUANG-ZHONG GAO ${ }^{1 *}$ and SHI-MING ZHANG ${ }^{2}$ \\ ${ }^{1}$ Department of Neurosurgery, Taizhou People's Hospital, Taizhou, Jiangsu 225300; ${ }^{2}$ Department of Neurosurgery, \\ The First Affiliated Hospital of Soochow University, Suzhou, Jiangsu 215006, P.R. China
}

Received April 14, 2014; Accepted January 8, 2015

DOI: $10.3892 / \mathrm{ol} .2015 .2904$

\begin{abstract}
Malignant gliomas are the most common primary brain tumors in adults and are associated with the highest mortality rate. Glioma invasion is one of the most notable causes of the poor prognosis of this cancer. Preventing the invasive behavior of malignant glioma cells by altering effector molecules can significantly improve the prognosis of a patient. microRNAs (miRNAs) are small noncoding RNAs, 22 nucleotides in length, that are able to function as oncogenes or tumor suppressors in human cancer. In the present study, the expression level of miRNA 218 (miR-218) was found to be markedly downregulated in glioma cell lines and human primary glioma tissues. miR-218 upregulation was found to dramatically reduce the migratory speed and invasive ability of glioma cells. Furthermore, it was demonstrated that ectopic expression of miR-218 in glioma cells resulted in the downregulation of roundabout, axon guidance receptor, homolog 1 (Robo1), upregulation of Slit homolog 2 (Slit2) and the expression of associated proteins following Robol knockdown by small interfering RNA. In addition, it was demonstrated that miR-218 inactivated the Slit2-Robo1 pathway through downregulating Robol expression by directly targeting the 3'-untranslated region (3'-UTR) of Robo1. The present results indicate that miR-218 plays important roles in preventing the invasiveness of glioma cells, and reveals a novel mechanism of miRNA-mediated direct suppression of the Slit2-Robol pathway in glioma.
\end{abstract}

\section{Introduction}

Human gliomas originate from neural mesenchymal cells and account for $4-50 \%$ of nervous system tumors (1). The

Correspondence to: Mr. Jian-Jun Gu, Department of Neurosurgery, Taizhou People's Hospital, 210 Yingchun Road, Taizhou, Jiangsu 225300, P.R. China

E-mail: gujianjundt@163.com

${ }^{*}$ Contributed equally

Key words: miR-218, gliomas, invasiveness, Slit2, Robo1
2007 World Health Organization (WHO) classification (2) classifies astrocytomas as well-differentiated low-grade diffuse astrocytoma (WHO grade I-II), anaplastic astrocytoma (WHO grade III) and glioblastoma multiforme (GBM; WHO grade IV). Despite the use of aggressive surgery combined with radiation, chemotherapy and biological therapy (3), glioma remains a notable therapeutic challenge. There is an acknowledged requirement for novel therapeutic approaches based on an increased understanding of the biological and molecular nature of glioma (4).

microRNAs (miRNAs) are a class of short, non-coding single-stranded RNA molecules that are 22-25 nucleotides in length. miRNAs negatively regulate gene expression through the post-transcriptional silencing of target messenger RNAs (mRNAs), which occurs due to complementary binding $(5,6)$. An increasing quantity of evidence has indicated an important role for miRNAs in the development of various cancers, including gliomas, and miRNAs have been associated with tumor suppressor and oncogenic activities $(7,8)$. Among these miRNAs, miRNA 218 (miR-218) has been demonstrated to be downregulated in human GBM specimens compared with the adjacent tumor-free brain tissue (9-12). Accumulated evidence has revealed that upregulation of miR-218 can inhibit tumor cell invasion and proliferation in glioma cells by altering the expression of multiple target genes (12-15).

Previous studies have found that miR-218 inhibited the invasion and metastasis of gastric cancer by targeting the roundabout, axon guidance receptor, homolog 1 (Robo1) receptor and suppressing nasopharyngeal cancer progression through the downregulation of survivin and the Slit homolog 2 (Slit2)-Robol pathway $(16,17)$. In the present study we examined how miR-218 affects the migration and invasion of glioma cells and the mechanism for miRNA-mediated direct suppression of the Slit2-Robol pathway in gliomas.

\section{Materials and methods}

Clinical samples. Tumor specimens were obtained from patients who underwent positive debulking surgery in the Neurosurgery Department of the The First Affiliated Hospital of Soochow University (Suzhou, China) between 2011 and 2013. The diagnosed gliomas were reviewed by an experienced neuropathologist, using histological slides, according to the 2007 WHO classification. The present study comprised 
20 grade I-II, 20 grade III and 20 grade IV glioma samples. In addition, 10 normal brain tissue samples were obtained from internal decompression of patients with cerebral injury. This study complied with the requirements of the ethics committee of The First Affiliated Hospital of Soochow University and informed consent was obtained from all participants.

Cell lines and transfection. Primary normal human astrocytes (NHA) were purchased from Sciencell Research Laboratories (Carlsbad, CA, USA). The U251, U87, SNB19 and LN229 glioma cell lines were obtained from the Institute of Biochemistry and Cell Biology (Shanghai Institutes for Biological Sciences, Chinese Academy of Science, Shanghai, China). The cells were maintained in RPMI-1640 medium containing $10 \% \mathrm{FBS}, 50$ units $/ \mathrm{ml}$ penicillin $\mathrm{G}$, and $250 \mu \mathrm{g} / \mathrm{ml}$ streptomycin (all purchased from Invitrogen Life Technologies, Carlsbad, CA, USA) in a humidified atmosphere containing $5 \% \mathrm{CO}_{2}$, at $37^{\circ} \mathrm{C}$. Transfections with miR-218 were performed in serum-free medium $24 \mathrm{~h}$ subsequent to plating, using Lipofectamine 2000 (Invitrogen Life Technologies). After $6 \mathrm{~h}$, the cells were placed in complete medium and maintained at $37^{\circ} \mathrm{C}$ in a $5 \% \mathrm{CO}_{2}$ atmosphere.

Small interfering (si)RNA and transfection assays. Robol-specific siRNA (forward, 5'-GGAUGUAUUUGCAAC AAGATT-3' and reverse, 5'-UCUUGUUGCAAAUACAUC CTT-3') was chemically synthesized by Qiagen (Hilden, Germany). The U87 cells were transfected with siRNA (HiPerFect Transfection Reagent; Qiagen) according to the manufacturer's instructions. Briefly, the original stock of the siRNA was suspended in the siRNA suspension buffer provided by the manufacturer and stored at $-20^{\circ} \mathrm{C}$ until use. On the day of transfection, $1 \times 10^{5}$ cells were seeded in six-well plates (Corning Inc., Corning, NY, USA) with a total volume of $1,000 \mu \mathrm{l}$ per well. siRNA was then gently introduced into the cells by adding $4 \mu$ l Oligofectamine ${ }^{\mathrm{TM}}$ Transfection Reagent (Invitrogen Life Technologies) and $5 \mu 1$ siRNA (20 nM) per well. Non-silencing siRNA (GE Dharmacon, Lafayette, CO, USA) (forward, 5'-UUCUCCGAACGUGUCACGUTT-3' and reverse, 5'-ACGUGACACGUUCGGAGAATT-3') was used to control any effects of the transfection reagent and siRNA. The in vitro assays described herein were performed $24 \mathrm{~h}$ after transfection.

RNA extraction and reverse transcription-quantitative polymerase chain reaction ( $R T-q P C R)$. Total RNA, including miRNA, was extracted from the cultured cells or fresh glioma cancer tissues using TRIzol reagent (Invitrogen Life Technologies), according to the manufacturer's instructions. The expression level of miR-218 was quantified using an miRNA-specific TaqMan miRNA Assay kit (Applied Biosystems, Foster City, CA, USA). U6 small nuclear RNA was used as an internal control. The mRNA expression of Slit2 and Robol was analyzed by qPCR, using the SYBR-Green method. All protocols were performed according to the manufacturer's instructions and the results were normalized to the expression of GAPDH. The primer sequences were as follows: Slit2 forward, 5'-ACCTCT TGGCCAATCCTTTT-3' and reverse, 5'-GAAGTCCTGAAT GGCCACAT-3'; Robol forward, 5'-GCCACCAGCAAGGAT GTATT-3' and reverse, 5'-CCTGTAACATGGGCTGGAGT-3'; and GAPDH forward, 5'-TCGGAGTCAACGGATTTGG-3' and reverse, 5'-CATGGGTGGAATCATATTGGA-3'.

Western blotting. The cells were lysed in 1\% Nonidet P-40 lysis buffer for 48 h following exposure to LY294002 or vehicle treatment. The homogenates were clarified by centrifugation at $20,000 \mathrm{xg}$ for $15 \mathrm{~min}$, at $4^{\circ} \mathrm{C}$, and the protein concentrations were determined with a bicinchoninic acid protein assay kit (Pierce Biotechnology, Inc., Rockford, IL, USA). SDS-PAGE was performed on $40 \mu \mathrm{g}$ of protein from each sample, the gels were transferred to polyvinylidene fluoride membranes (Merck Millipore, Darmstadt, Germany) and incubated with primary polyclonal goat anti-human Slit2 and rabbit anti-human Robol antibodies (1:200 dilution; sc-26599 and sc-25672, respectively, Santa Cruz Biotechnology, Inc., Dallas, TX, USA) followed by incubation with horseradish peroxidase-conjugated monoclonal goat anti-rabbit and donkey anti-goat secondary antibodies (1:1,000 dilution; cat nos. sc-2004 and sc-2020, respectively; Zymed Life Technologies, Carlsbad, CA, USA). The membranes were stripped using $1 \mathrm{X}$ phosphate-buffered saline with Tween 20 buffer (Wuhan Boster Biological Technology, Ltd., Wuhan, China) and reprobed with a primary monoclonal mouse anti-rabbit antibody against GAPDH (1:1,000 dilution; Bioworld, Nanjing, China). The protein bands were quantitated by densitometry using the gel analysis software ImageJ (National Institutes of Health, Bethesda, MA, USA. The values were normalized to GAPDH expression.

Transwell assay and scratch-wound assay. Cell invasion was determined using Transwell and scratch-wound assays. For the Transwell assay, the appropriate oligonucleotides were transfected into the cells according to the aforementioned protocol. Subsequent to incubation for $48 \mathrm{~h}, 3 \times 10^{4}$ cells were transferred to the top of the Matrigel-coated invasion chambers (BD Biosciences, San Jose, CA, USA) in a serum-free Dulbecco's modified Eagle's medium (DMEM). DMEM containing $10 \%$ fetal bovine serum was added to the lower chamber. After $24 \mathrm{~h}$, the non-invading cells were removed, and the invading cells were fixed using $95 \%$ ethanol, stained with $0.1 \%$ crystal violet and images were captured under a x100 magnification. The tests were repeated in three independent experiments. For the scratch-wound assay, the appropriate oligonucleotides were transfected into the cells in six-well plates. The cell layers were then scratched using a $200 \mu \mathrm{l}$ sterile pipette tip to form wound gaps. The wound location in the six-well plates was marked. Images of the cells were captured to record the wound width at 0 and $24 \mathrm{~h}$, and the images were captured at the marked wound location to measure the migratory ability of the cells.

Luciferase assay. The 3'-UTR sequence of Robol predicted to interact with miR-218 or a mutated sequence with the predicted target sites was synthesized and inserted into the $\mathrm{XbaI}$ and FseI sites of a pGL3 control vector (Promega Corporation, Madison, WI, USA). These constructs were named pGL3-Robo1-3'-UTR and pGL3-Robo1-3'-UTR-mut, respectively. For the reporter assay, the U87 cells were plated onto 24-well plates and transfected with pGL3-Robo1-3'-UTR or pGL3-Robo1-3'-UTR-mut and P-miR-218 or P-miR-control 

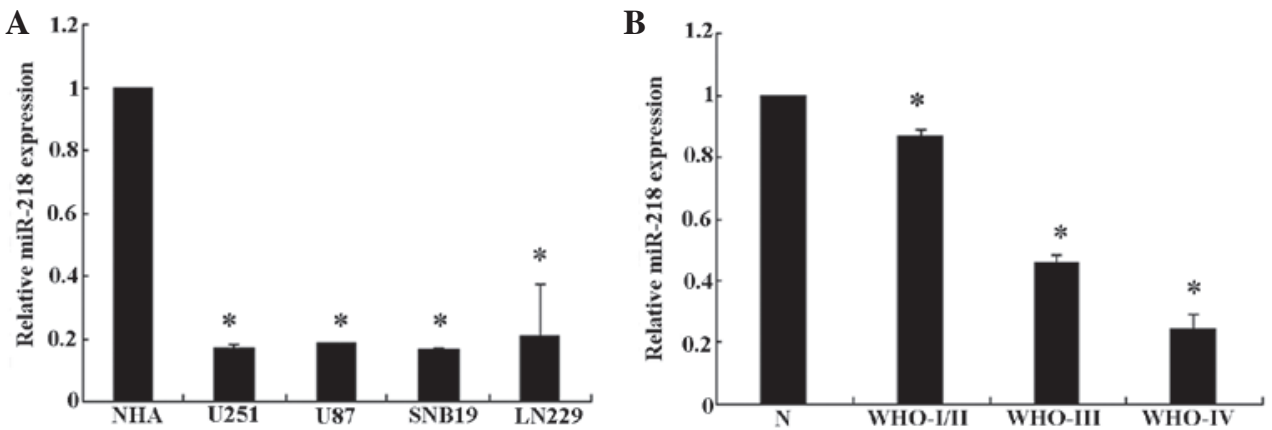

Figure 1. miR-218 expression in glioma cells and tissues. (A) miR-218 expression was detected in the glioma U251, U87, SNB19 and LN229 and primary NHA cell lines. qPCR demonstrated that miR-218 was downregulated in all of the glioma cell lines compared with the NHA cells. ${ }^{*} \mathrm{P}<0.05$ vs. NHA cells. (B) miR-218 was detected in 10 normal brain and 60 glioma tissues by qPCR. miR-218 expression was significantly lower in glioma tissues compared with normal brain tissue. "P<0.05 vs. normal brain tissue. miR-218, microRNA 218; NHA, normal human astrocytes; WHO, World Health Organization; qPCR, quantitative polymerase chain reaction.

vectors using the FuGENE HD transfection reagent (Promega Corporation). A Renilla luciferase vector pRL-SV50 (Promega Corporation) was cotransfected to normalize the differences in transfection efficiency. Subsequent to $48 \mathrm{~h}$ transfection, the cells were harvested and assayed using the Dual-Luciferase Reporter Assay System (Promega Corporation), according to the manufacturer's instructions. Transfection was repeated in triplicate in three independent experiments.

Target prediction and network analysis. Robol was identified as a notable novel target of miR-218 using the conventional prediction tool, TargetScan (www.targetscan.org; Whitehead Institute for Biomedical Research, Cambridge, MA, USA).

Statistical analysis. Experimental data were presented as the mean \pm standard deviation. All analyses were performed using a two-tailed Student's $t$-test performed on SPSS software, version 12.0 (SPSS, Inc., Chicago, IL, USA). $\mathrm{P}<0.05$ was considered to indicate a statistically significant difference.

\section{Results}

miR-218 is downregulated in glioma tissues. To analyze the expression levels of miR-218, a qPCR analysis of miR-218 expression was conducted in the U251, U87, SNB19 and LN229 glioma cell lines. The present results revealed that miR-218 is downregulated in all glioma cell lines compared with the NHA cells $(\mathrm{P}<0.05)$. In addition, when miR-218 expression was measured in 10 normal brain tissue and 60 glioma tissue samples, it was observed that the expression level of miR-218 was significantly decreased in the glioma tissues, particularly in grade III/IV glioma tissues, compared with the normal brain tissues (Fig. 1). This result demonstrates that miR-218 expression decreases markedly between normal brain tissue and low grade to GBM tissue. Overall, the present results indicate that miR-218 is downregulated in glioma cell lines and glioma tissues.

Upregulation of miR-218 inhibited cell invasion in U87 cells. As invasiveness is one of the pathophysiological features of human malignant gliomas, the effects of miR-218 on the invasiveness and migration of glioma cells were checked by Transwell and scratch-wound assays. An in vitro Matrigel invasion assay revealed that the invasiveness of U87 cells transfected with the miR-218 mimic was suppressed compared with control and NC groups (Fig. 2A and B). The results of the in vitro wound healing assay revealed that miR-218 significantly attenuated the migration of U87 cells compared with control and NC groups (Fig. 2C and D). This finding indicates that the upregulation of miR-218 inhibits the invasive ability of glioma cells in vitro.

Upregulation of miR-218 reduces Robol expression via the inactivation of Slit2-Robol signaling. Development of invasiveness by malignant glioma cells involves multiple genetic alterations in signaling pathways. Numerous studies have reported that the Slit2-Robol signaling channels can inhibit glioma invasion and migration. However, the specific roles of Slit2/Robol in cancer cell invasion have not yet been completely elucidated in vivo. The present study also observed, using qPCR and western blot analysis, that treatment with the miR-218 mimic for $24 \mathrm{~h}$ significantly upregulated the expression of Slit2, which was followed by a decrease in Robol (Fig. 3). These results indicate that miR-218 reduced the expression of Robol via the inactivation of Slit2-Robo1 signaling.

Robol is a functional downstream target of miR-218. A luciferase reporter assay further confirmed the direct interaction between miR-218 and the 3'-UTR of Robol mRNA (Fig. 4). The luciferase activity for the wild-type 3'-UTR of Robol was significantly inhibited by co-transfection with miR-218 mimics compared with constructs containing mutated 3'-UTRs. The present study demonstrated that Robol is a direct target of $\operatorname{miR}-218$.

Robol siRNA can imitate the role of miR-218 in U87 glioma cells. To assess the role of Robol in the miR-218-dependent inhibition of cell migration and invasion, miR-218 inhibitor was transfected into U87 cells treated with Robol siRNA. As expected, Robol protein expression was significantly reduced by the specific Robol siRNA (Fig. 5). The enhanced invasive ability of miR-218 inhibitor-transfected U87 cells declined when Robol siRNA was co-transfected with the miR-218 inhibitor (Fig. 6). These results indicate that Robol is essential for miR-218-dependent cell migration and invasion. 
A

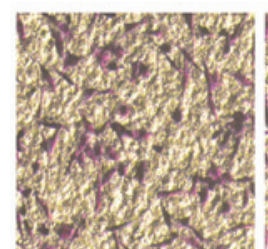

Control

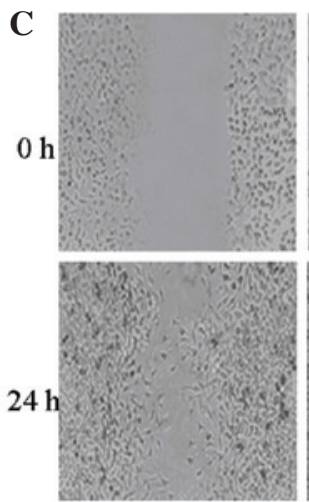

Control

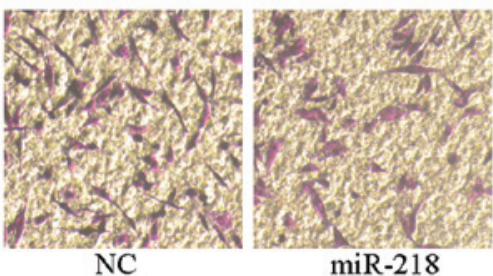

miR-218

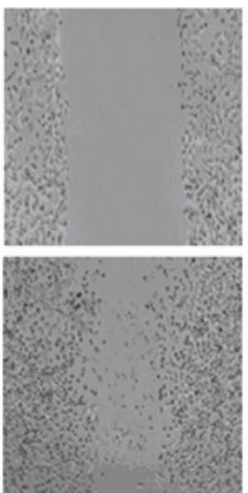

NC

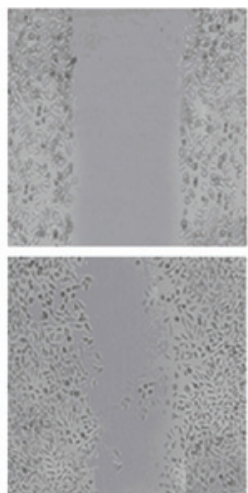

$\operatorname{miR}-218$

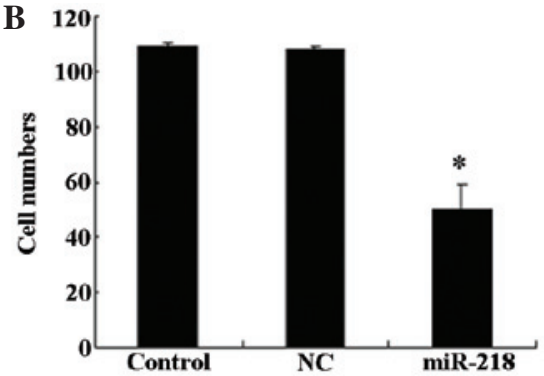

D

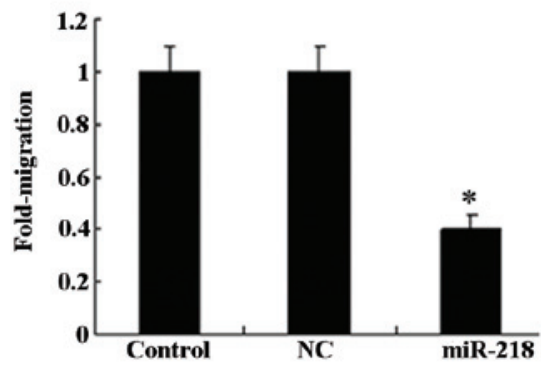

Figure 2. miR-218 suppresses U87 cell invasion. (A and B) U87 cells were examined for cell invasion in 24-well plates with Transwell chambers. The migrated cells were stained with crystal violet. The invasiveness of U87 cells was attenuated with the upregulation of miR-218. (C and D) Images of the scratch-wound healing assay were captured at 0 and $24 \mathrm{~h}$, and the wound gaps were analyzed by measuring the distance of migrating cells for three regions of each wound. ${ }^{*} \mathrm{P}<0.05$. NC, negative control; miR-218, microRNA 218.
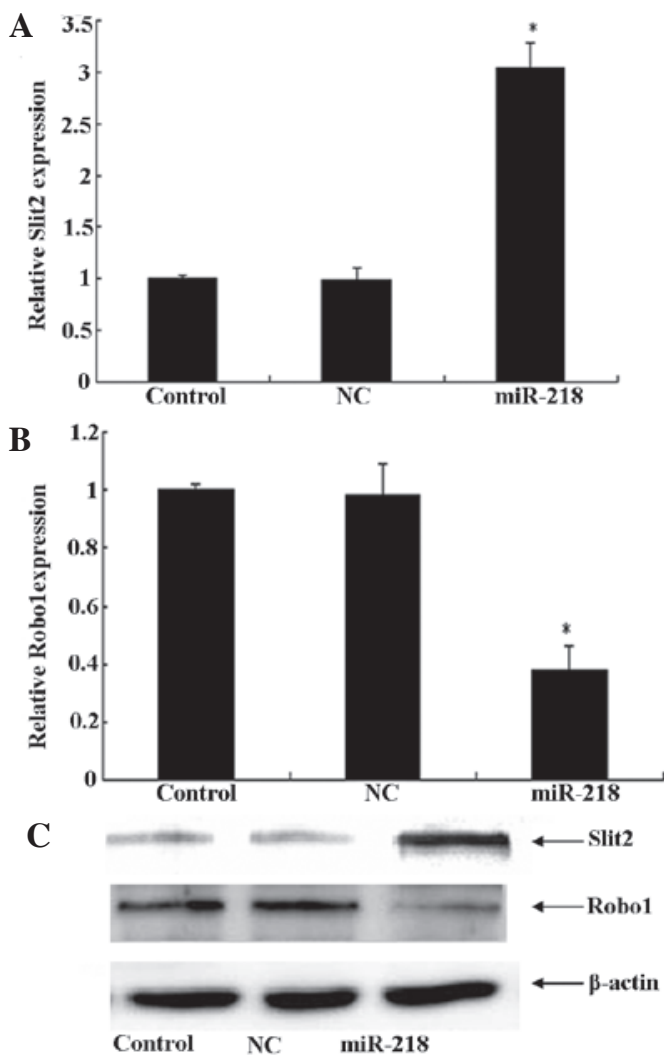

Figure 3. Upregulation of miR-218 reduces Robo1 expression through the inactivation of Slit2-Robo1 signaling. (A and B) The U87 cells were treated with control, $\mathrm{NC}$ and miR-218 mimics, and the levels of Robol and Slit2 mRNA were measured by quantitative polymerase chain reaction. (C) The level of Robo1 and Slit2 protein expression in the U87 cells was measured by western blot analysis. ${ }^{*} \mathrm{P}<0.05$. Robo 1 , roundabout, axon guidance receptor, homolog 1; Slit2, Slit homolog 2; NC, negative control; miR-218, microRNA 218.
A Human Robo1 3'UTR UUUUUUUGGUUUUAA -AAGCACAA...

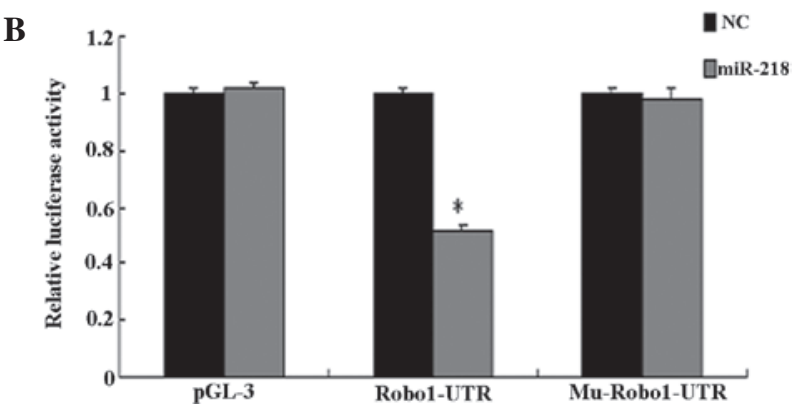

Figure 4. Robol is a direct target of miR-218. (A) Identification of target sites in the 3'-UTR of Robol was performed using TargetScan. (B) A luciferase assay was performed to determine the target association in U87 cells transfected with the control pGL-3 vector, a vector containing the Robol sequence that was predicted to interact with miR-218 (Robol-UTR) or a vector containing a mutated Robol sequence with the predicted target sites (Mu-Robol-UTR) . The reported data are representative of at least three independent experiments. ${ }^{*} \mathrm{P}<0.05$. Robo 1 , roundabout, axon guidance receptor, homolog 1; UTR, untranslated region; miR-218, microRNA 218; $\mathrm{NC}$, negative control.

\section{Discussion}

Malignant gliomas are diffuse tumors that are extremely invasive and usually multifocal. Glioma possesses a dismal prognosis, with a median survival of $\sim 16$ months (18). The ability for single tumor cell infiltration, which involves the extension of tendrils of the tumor several centimeters away 


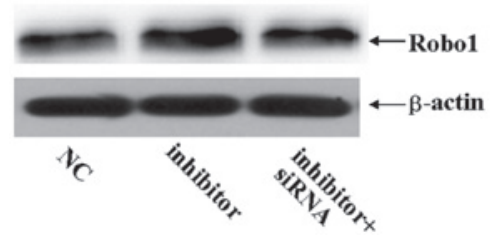

Figure 5. Western blot analysis revealed that the protein expression of Robol was significantly increased in miR-218 inhibitor-transfected U87 glioma cells. By contrast, Robo1 siRNA decreased the miR-218 inhibitor-induced increase in Robol protein levels. ${ }^{*} \mathrm{P}<0.05$. Robo 1, roundabout, axon guidance receptor, homolog 1; NC, negative control; siRNA, small interfering RNA; miR-218, micro RNA 218.
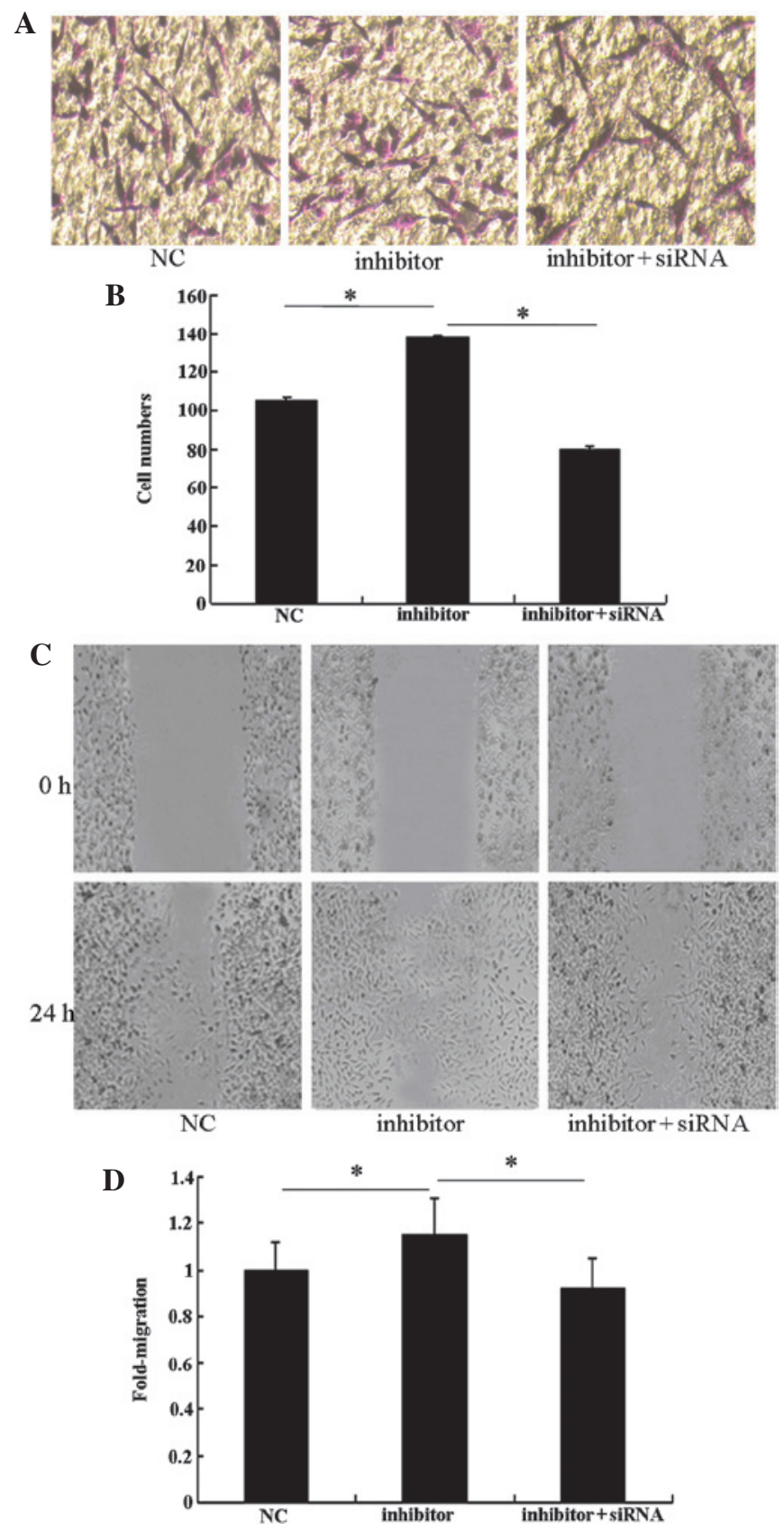

Figure 6.In the U87 cell line, Robol siRNA reduced miR-218 inhibitor-induced enhanced invasive ability. (A and B) Transwell assays revealed that the miR-218 inhibitor promoted the invasion of U87 cells and Robo1 siRNA attenuated the invasion. "P<0.05. (C and D) Robol siRNA attenuation of the migration of U87 cells was also observed in the scratch-wound assay. ${ }^{*} \mathrm{P}<0.05$. NC, negative control; siRNA, small interfering RNA; Robo1, roundabout, axon guidance receptor, homolog 1; miR-218, microRNA 218 from the main tumor mass, is one of the major obstacles for the effective treatment of gliomas, as this infiltration results in incomplete surgical removal and contributes to the high frequency of tumor recurrence (19). Despite the increasing quantity of evidence that demonstrates the promotion of glioma cell infiltration into the brain parenchyma by various stimuli, the mechanisms underlying the dysregulation of cell motility during tumor invasion by the insidious glioma cells have yet to be elucidated (20). As a result, exploring novel treatment methods is an urgent clinical challenge for neuroscientists.

Previous studies have revealed that miR-218 expression is often downregulated in several human cancers, including gastric cancer, lung squamous cell carcinoma, malignant astrocytomas and medulloblastomas, which indicates that miR-218 may function as a tumor suppressor (21-23). Studies have identified that the ectopic expression of miR-218 contributes to the inhibition of proliferation, invasion and migration in glioma cells, as well as the induction of apoptosis by downregulating the gene that was directly targeted by miR-218 (12-15). Firstly, miR-218 inhibits the expression of the target gene inhibitor of nuclear factor $\kappa \mathrm{B}(\mathrm{NF}-\kappa \mathrm{B})$ kinase $\beta$, and in a dose-dependent manner, inhibits the expression of NF- $\kappa B$, whilst reducing the expression of matrix metalloproteinase (MMP) 9 and inhibiting the invasion and migration ability of glioma cells (12). Secondly, epidermal growth factor receptor-coamplified and overexpressed protein (ECOP) has been identified as a functional downstream target gene of miR-218 that can regulate NF- $\mathrm{\kappa B}$ transcription activity and is associated with the apoptotic response. Overexpression of miR-218 can restrain the activity of NF- $\mathrm{KB}$ through ECOP, thus inducing glioma cell apoptosis and inhibiting the activity, proliferation and tumorigenicity of glioma cells (13). Thirdly, the expression of lymphoid enhancer-binding factor 1 (LEF1) and MMP-9 in the high grade glioma group is extremely high, while the expression in the low-grade glioma group is extremely low, and is negatively correlated with the expression of miR-218. Overexpression of miR-218 inhibits the Wnt/LEF1 signaling pathways that lead to a reduction in MMP-9 synthesis, inhibiting tumor invasion (14). Finally, the abnormal expression of miR-218 in glioma cells decreased, but there was an abnormal increase in cyclin-dependent kinase (CDK) 6 expression, with the expression level of the two being negatively correlated. Overexpression of miR-218 in the glioma cell lines can inhibit CDK6 expression and glioma cell proliferation and promote its apoptosis (15).

Tumor development is a complex multi-step process that includes malignant tumor invasion and metastasis (24). The development of invasiveness in malignant glioma cells involves several genetic alterations within signaling pathways. Numerous studies have reported that the Slit2/Robol signaling channels can inhibit glioma invasion and migration. An in vitro study performed by Mertsch et al (25) identified, using a modified Boyden chamber assay, that the Slit2/Robo1 system serves as a chemorepellent for glioma cells, indicating that glioblastoma cells migrate away from increased Slit2 concentrations and prompt Robol-positive glioma cell invasion along gray matter tracts and into white matter, including the corpus callosum (25). A previous in vivo study revealed that ectopic expression of Slit2 in SNB19 cells attenuates cell migration and invasion (20). These results indicate that Slit2/Robol can inhibit 
glioma invasion and migration in vivo and in vitro. Slit2 may also be a tumor suppressor gene that inhibits the migration and invasion of tumor cells and this inhibition appears to be mediated by Robol (20). Previous studies identified that miR-218 inhibited invasion and metastasis of gastric cancer by targeting the Robol receptor and suppressed nasopharyngeal cancer progression through the downregulation of survivin and the Slit2-Robo1 pathway $(16,17)$.

In the present study, Robol was identified as a notable novel target of miR-218 using the conventional prediction tool TargetScan (www.targetscan.org). The expression levels of miR-218, Robol and Slit2 were detected in 70 tissue samples, consisting of normal brain tissue and low- and high-grade glioma tissues, using RT-qPCR and western blot analysis. It was found that the expression of miR-218 and Slit2 was always inverse to that of Robol. Notably, the mRNA and protein levels of Robol were significantly decreased and the mRNA and protein levels of Slit2 were significantly increased subsequent to the transfection of miR-218 mimics into U87 cells. Furthermore, it was found that miR-218 was involved in modulation of the Slit2-Robol signaling pathway and downregulation of Robol expression by directly targeting the Robol 3'-UTR. In addition, Robol siRNA can reduce the invasive ability of the cells subsequent to its enhancement by the exogenous expression of the miR-218 inhibitor. Overall, the present results indicate that miR-218 inhibits the migration and invasion of glioma cells through the Slit2-Robol signaling pathway. Therefore, the development miR-218 as a biomarker for glioma or as a potential therapeutic candidate for miRNA replacement therapy is extremely promising $(26,27)$.

\section{References}

1. Dallol A, Krex D, Hesson L, Eng C, Maher ER and Latif F: Frequent epigenetic inactivation of the SLIT2 gene in gliomas. Oncogene 22: 4611-4616, 2003.

2. Louis DN, Ohgaki H, Wiestler OD, et al: The 2007 WHO classification of tumours of the central nervous system. Acta Neuropathol 114: 97-109, 2007.

3. Grauer OM, Wesseling P and Adema GJ: Immunotherapy of diffuse gliomas: biological background, current status and future development. Brain Pathol 19: 674-693, 2009.

4. Dontula R, Dinasarapu A, Chetty C, et al: MicroRNA 203 Modulates Glioma Cell Migration via Robo1/ERK/MMP-9 Signaling. Genes Cancer 4: 285-296, 2013.

5. Bartel DP: MicroRNAs: target recognition and regulatory functions. Cell 136: 215-233, 2009.

6. Long JM and Lahiri DK: Advances in microRNA experimental approaches to study physiological regulation of gene products implicated in CNS disorders. Exp Neurol 235: 402-418, 2012.

7. Park S and James CD: ECop (EGFR-coamplified and overexpressed protein), a novel protein, regulates NF-kappaB transcriptional activity and associated apoptotic response in an IkappaBalpha-dependent manner. Oncogene 24: 2495-2502, 2005.
8. Xu B, Hsu PK, Karayiorgou M and Gogos JA: MicroRNA dysregulation in neuropsychiatric disorders and cognitive dysfunction. Neurobiol Dis 46: 291-301, 2012.

9. Godlewski J, Nowicki MO, Bronisz A, Williams S, et al: Targeting of the Bmi-1 oncogene/stem cell renewal factor by microRNA-128 inhibits glioma proliferation and self-renewal. Cancer Res 68: 9125-9130, 2008.

10. Silber J, Lim DA, Petritsch C, et al: MiR-124 and miR-137 inhibit proliferation of glioblastoma multiforme cells and induce differentiation of brain tumor stem cells. BMC Med 6: 14, 2008.

11. Rao SA, Santosh V and Somasundaram K: Genome-wide expression profiling identifies deregulated miRNAs in malignant astrocytoma. Mod Pathol 23: 1404-1417, 2010.

12. Song L, Huang Q, Chen K, et al: miR-218 inhibits the invasive ability of glioma cells by direct downregulation of IKK- $\beta$. Biochem Biophys Res Commun 402: 135-140, 2010.

13. Xia H, Yan Y, Hu M, et al: MiR-218 sensitizes glioma cells to apoptosis and inhibits tumorigenicity by regulating ECOP-mediated suppression of NF- $\kappa \mathrm{B}$ activity. Neuro Oncol 15: 413-422, 2013.

14. Liu Y, Yan W, Zhang W, et al: MiR-218 reverses high invasiveness of glioblastoma cells by targeting the oncogenic transcription factor LEF1. Oncol Rep 28: 1013-1021, 2012.

15. Zhang JM, Sun CY, Yu SZ, et al: Relationship between miR-218 and CDK6 expression and their biological impact on glioma cell proliferation and apoptosis. Zhonghua Bing Li Xue Za Zhi 40: 454-459, 2011 (In Chinese).

16. Tie J, Pan Y, Zhao L, et al: MiR-218 inhibits invasion and metastasis of gastric cancer by targeting the Robol receptor. PLoS Genet 6: e1000879, 2010.

17. Alajez NM, Lenarduzzi M, Ito E, et al: MiR-218 suppresses nasopharyngeal cancer progression through downregulation of survivin and the SLIT2-ROBO1 pathway. Cancer Res 71: 2381-2391, 2011

18. Stupp R, Mason WP, van den Bent MJ, et al; European Organisation for Research and Treatment of Cancer Brain Tumor and Radiotherapy Groups; National Cancer Institute of Canada Clinical Trials Group: Radiotherapy plus concomitant and adjuvant temozolomide for glioblastoma. N Engl J Med 352: 987-996, 2005.

19. Furnari FB, Fenton T, Bachoo RM, et al: Malignant astrocytic glioma: genetics, biology, and paths to treatment. Genes Dev 21: 2683-2710, 2007.

20. Yiin JJ, Hu B, Jarzynka MJ, et al: Slit2 inhibits glioma cell invasion in the brain by suppression of Cdc 42 activity. Neuro Oncol 11: 779-789, 2009.

21. Martinez I, Gardiner AS, Board KF, et al: Human papillomavirus type 16 reduces the expression of microRNA-218 in cervical carcinoma cells. Oncogene 27: 2575-2582, 2008.

22. Petrocca F, Visone R, Onelli MR, et al: E2F1-regulated microRNAs impair TGFbeta-dependent cell-cycle arrest and apoptosisin gastric cancer. Cancer Cell 13: 272-286, 2008.

23. Yanaihara N, Caplen N, Bowman E, et al: Unique microRNA molecular profiles in lung cancer diagnosis and prognosis. Cancer Cell 9: 189-198, 2006.

24. Xu Y, Li WL, Fu L, et al: Slit2/Robo1 signaling in glioma migration and invasion. Neurosci Bull 26: 474-478, 2010.

25. Mertsch S, Schmitz N, Jeibmann A, et al: Slit2 involvement in glioma cell migration is mediated by Robol receptor. J Neurooncol 87: 1-7, 2008.

26. Bader AG, Brown D and Winkler M: The promise of microRNA replacement therapy. Cancer Res 70: 7027-7030, 2010.

27. Roth P, Wischhusen J, Happold C, et al: A specific miRNA signature in the peripheral blood of glioblastoma patients. J Neurochem 118: 449-457, 2011. 